\title{
FIV Energy Harvesting from Sharp Edge Square and Diamond Oscillators
}

\section{Vahid Tamimi ${ }^{*}$, Mohammad Said Seif ${ }^{2}$, Selda Shahvaghar-Asl ${ }^{3}$, Seyed Taghi Omid Naeeni ${ }^{4}$, Mostafa Zeinoddini ${ }^{5}$}

I Ph.D., School of Civil Engineering, College of Engineering, University of Tehran, Tehran, Iran; vahid.tamimi@ut.ac.ir

${ }^{2}$ Professor, Department of Mechanical Engineering, Sharif University of Technology, Tehran, Iran; seif@ sharif.edu ${ }^{3}$ Ph.D. Student, Department of Civil Engineering, Sharif University of Technology, Tehran, Iran

4 Professor, School of Civil Engineering, College of Engineering, University of Tehran, Tehran, Iran; stnaeeni@ut.ac.ir

5 Professor, Department of Civil Engineering, K.N.Toosi University of Technology, Tehran, Iran; zeinoddini@kntu.ac.ir

\section{ARTICLE INFO}

\section{Article History:}

Received: 13 Jan. 2019

Accepted: 15 Jun. 2019

\section{Keywords:}

FIV energy harvesting

Hydroelastic efficiency

VIV

Galloping

Square and diamond cylinders

\begin{abstract}
The horizontal kinetic energy of the fluid flow, from on-land wind to ocean tidal stream, is one of the most promising sources of the energy. In the field of renewable energies, power extraction from Flow Induced Vibration (FIV) of bluff bodies is a fast growing research area which has seen a great advancement over the last decade. In this study, the FIV energy harvesting potential of a sharp edge square cylinder in two different flow incidences is investigated. The square cylinder, depending on its orientation with respect to the incident flow, demonstrates VIV or galloping types of responses. The results indicate that the square cylinder with a flat side perpendicular to the flow has a galloping type of response. In contrast, the same cylinder with a sharp vertex pointing the flow (diamond configuration) shows a VIV type of response. The hydroelastic efficiency of the resonating square cylinder is significantly higher than that with the galloping type of response. This shows the great advantages of diamond VIV excavators over square galloping harvesters.
\end{abstract}

\section{Introduction}

Flow Induced Vibration (FIV) remains a practical challenge in many disciplines such as civil, mechanical, ocean and aerospace engineering. Vortex Induced Vibration (VIV) and galloping are two main subcategories of the FIV phenomena. VIV is a resonant type of response in which the frequency of the vortices shed behind the bluff body locks-in with the natural frequency of the system and produces high amplitude oscillations. This is, however, a self-contained phenomenon, thus the amplitude of vibrations hardly exceeds one equivalent diameter [1]. Galloping is a classical type of instability, which refers to a low frequency type of cross-flow oscillation. It is characterized by large amplitude oscillations at high reduced velocities. According to Blevins [2], the amplitude of galloping may exceed several times of the equivalent diameter.

The cross-section shape, the after body profile and the flow incidence angle play key roles in the FIV mechanism and its subcategory. A circular cylinder, as a standard round section, undergoes cross-flow VIV when is freely oscillating in a uniform flow. The oscillations are self-limited because excessively large oscillations make the wake modes and the shedding patterns [3]. Large oscillations occur only at a certain range of reduced velocities, referred to as the lock-in range. The VIV in circular cylinders is hardly affected by variations in the flow incidence angle due to their axisymmetric cross-section. Comprehensive reviews on the VIV of circular cylinders can be found in Bearman [4], Parkinson [5], Sarpkaya [6], Williamson and Govardhan [3] and Gabbai and Benaroya [7].

A typical non-circular sharp-edge bluff body is a square cylinder. Flow regimes, pressure fields, hydrodynamic forces, wake frequencies and FIV of square and rectangular cylinders were excessively studied by several researchers. Comprehensive reviews on the flow regimes around and FIV of square cylinders were provided in Blevins [8], Naudascher and Rockwell [9] and Païdoussis et al. [10]. It is noted that in-water experiments on square cylinders (with low massdamping parameters) are much less in number than those performed in air. Nemes et al. [11], Barrero and 
Fernandez [12], Zhao et al. [13] and Xu-Xu et al. [14] examined the influence of the flow incidence angle and mass ratio on the transverse response of square cylinders. They found that FIV of square cylinders was significantly affected by variations in the flow incidence angle. Square cylinders with low mass ratios, reportedly, demonstrated both VIV and galloping types of response. A galloping type of response was reported when a flat side stayed perpendicular to the flow so the amplitude of oscillations almost linearly increased by the increase in the flow velocity.

Flow regimes around cylinders with a diamond section were studied in wind tunnels by Obasaju et al. [15] and Dutta et al. [16]. The transverse oscillations of low mass ratio cylinders with a diamond section were experimentally investigated by Zhao et al. [13]. The experiments were conducted in a re-circulating water channel. They found that the diamond cylinders exhibit a VIV type of response but the VIV here was more complicated than that in a circular cylinder.

Due to the detrimental nature of the FIV, most of the previous studies are directed at understanding and control of this phenomenon. In the context of renewable energies, however, FIV is considered as one of the main sources of the mechanical energy harvesting. While most FIV energy harvesting methods are capable for low power energy excavation, some can be used for high power electricity production. VortexInduced Vibration Aquatic Clean Energy (VIVACE) [17] is one of the high power (as high as 1GW) energy harvesters that convert FIV of a bluff body into electricity. The original concept took advantage of VIV of a single circular cylinder for energy extraction [18]. Several modifications are done since the invention of VIVACE converter to increase its electromechanical or hydroelastic efficiencies. Enhancement of the hydroelastic efficiency has been done through geometrical modifications of circular bluff bodies by the use of Passive Turbulence Control (PTC) [19, 20, 21] or by their application in an array [22, 23]. Application of non-circular cross-sections with combined galloping and VIV responses can be regarded as an alternative approach for the enhancement of the converter efficiency.

Despite valuable researches have been done on the FIV of non-circular cylinders, the subject of FIV energy harvesting from sharp-edge cylinders has received little attention in the literature. Analytical studies have been done by Abdelkefi et al. [24] on the exploitation of electrical power from the galloping of the square and isosceles triangular prisms. Zhang et al. [25] experimentally investigated the energy transference from FIV of circular, square and regular triangular cylinders. They found that the circular and triangular prisms have better energy performance with respect to the square cross-section. Hemon et al. [26] had wind tunnel experiments on FIV energy harvesting of elastically mounted prisms by means of electromagnetic induction. The prisms had square and rectangular cross-sections. They reported that the efficiency of galloping harvesters is weak as compared to VIV oscillators.

The majority of the previous studies on the FIV energy harvesting are conducted in the air (with large massdamping parameters). Except for few studies on the rectangular prisms, other sharp-edge configurations like diamond cross-sections have been generally overlooked. The current in-water towing tank study deals with the FIV energy harvesting potential of square cylinders. The square cylinder is examined in two different symmetrical configurations with respect to the incident flow. The single degree of freedom vibrating system has a low mass-damping parameter $\left(m^{*} \zeta \approx 0.011\right)$ and the reduced velocity ranges from 1.6 to 24 .

\section{Experimental setup}

The current in-water experiments are carried out in the towing tank of the Marine Engineering Laboratory at the Sharif University of Technology (SUT). More information about the facilities can be found in $[27,28$, 29].

A linear elastic Single Degree Of Freedom (SDOF) support is positioned on top of the test cylinder. The support consists of two parallel horizontal acrylic slabs (each $15 \mathrm{~mm}$ thick). A pair of vertical thin spring-steel blade flexors is bolted in parallel to two sides of the horizontal slabs. The top slab is rigidly connected to the moving carriage. The test cylinder is vertically fixed by its upper end to the bottom slab of the elastic supporting device through a shear-beam load cell. The load cell follows the design used by Assi [30] and consists of two perpendicular shear webs that can simultaneously measure the lift and drag forces. The test arrangement enables the oscillating cylinder to vibrate as a rigid body in a single direction transverse to the flow. A view of the experimental setup for the cross-flow excited vibration experiments is shown in Figure 1(a).

In order to record the flow-induced responses of the downstream test cylinder, four strain gauges are positioned on the spring blades, adjacent to the regions of contra-curvature in a Wheatstone full bridge configuration. They enable monitoring the displacement response of the test cylinders. The supporting device is calibrated, before conducting each test series, by examining its load-displacement against the strain gauges data. The calibration tests demonstrate a linear elastic behavior for the supporting device in the loading and unloading cycles with no perceptible hysteresis. The stiffness of the linear elastic supporting device is calculated to be around $102 \mathrm{~N} / \mathrm{m}$. A horizontal circular end plate is attached underneath of the test cylinders to reduce the end effects. The end plate dimensions and thicknesses are chosen as per Morse et al. [31] recommendations. A distance of around $400 \mathrm{~mm}$ still remains between the end plates 
and the tank's bottom. In order to minimize the end plate interference on the incoming flow, the plate edges are neatly chamfered.

Test model used in the current study is made of acrylic (Figure 1(b)) and have a specific mass ratio and massdamping parameter. Table 1 provides a summary of the tests carried out on the square cross-section. It shows the square cylinder's configuration, the mass and damping ratio, the velocity range, and the Reynolds number extent in each of the test series. As it can be noticed, in the tests reported in the current paper the Reynolds number ranges from $2.3 \times 10^{3}$ to $4.0 \times 10^{4}$.

\section{Results and discussions}

\subsection{FIV response of the square cross-section}

The square cylinder is tested in two different configurations with respect to the incident flow. In order to validate the experimental arrangements and procedures used in the current study, the amplitude response of the cylinder is compared with the test results from Nemes et al. [11].

Figure 2(a) illustrates the cross-flow response of the diamond cylinder in the current study along with the test results from Nemes et al. [11]. In general, the figure indicates that the diamond cylinder in both studies exhibits a VIV type of response. There is also a reasonable agreement between the amplitude responses for the diamond cylinder in the current study and in Nemes et al. [11] experiments. However, the initial and upper branches and the onset of vibrations are slightly dissimilar in the two studies. Furthermore, the Nemes et al. [11] results show a broad lower branch. These can be attributed to differences in mass-damping parameters or mass ratios [30]. Nemes et al. [11] presented $A^{*}{ }_{10}$ (mean of top $10 \%$ of the peaks) while present data uses the RMS approach. For the present diamond cylinder, $A^{*}{ }_{10}$ of transverse responses are also given in Figure 2(a). As it can be noticed, with maximum transverse responses, the oscillations in the lower branch tend to continue beyond maximum velocity tested $\left(V_{r}=14.6\right)$.

The amplitude response in Figure 2(a) is characterized by a clear peak which appears at around $f_{s}=f_{N}$. This is similar to that with the classical circular cylinders. The onset of fluctuations in the diamond cylinder appears at $V_{r} \approx 2$, and the peak amplitude occurs at $V_{r} \approx 6.2$ or the corresponding vortex resonance velocity. The maximum amplitude in the diamond cylinder is $0.58 \mathrm{D}$. The amplitude response from the square configuration in the current study is presented in Figure 2(b) along with the test results from Nemes et al. [11] and $\mathrm{Xu}-\mathrm{Xu}$ et al. [14]. Figure 2(b) shows that the square cylinders in all studies clearly exhibit a galloping type of response. The oscillations start at $V_{r} \approx 4$. The reduced amplitude increases almost linearly with increase in the reduced velocity.

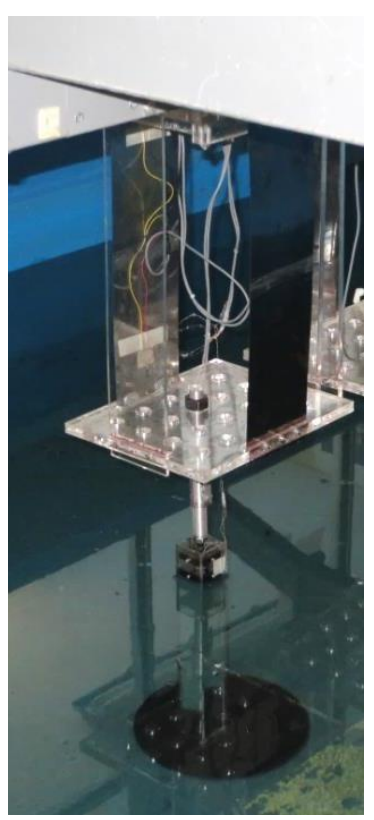

(a)

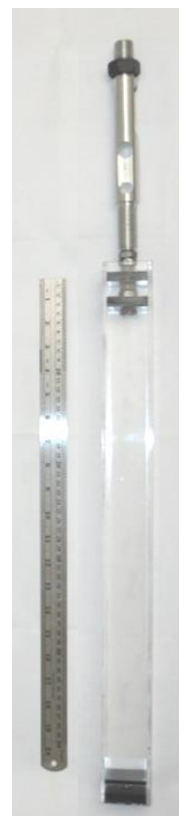

(b)

Figure 1. View of the experimental setup (a) and the square test model (b).

Table 1. Summary of the tests carried out on cylinders with different mass and damping ratios.

\begin{tabular}{|c|c|c|c|c|c|c|}
\hline Specimen & Configuration & $\mathrm{m}^{*}$ & $\begin{array}{c}\zeta \text { in water } \\
(\%)\end{array}$ & $\begin{array}{c}\zeta \text { in air } \\
(\%)\end{array}$ & $\begin{array}{c}\text { Velocity Range } \\
(\mathrm{m} / \mathrm{s})\end{array}$ & $\begin{array}{c}\text { Reynolds Number } \\
\text { Range }\end{array}$ \\
\hline Square cylinder & 专 & \multirow{2}{*}{1.69} & 5.39 & \multirow{2}{*}{0.65} & $0.053-0.76$ & $2,325-32,942$ \\
\hline Diamond cylinder & & & 5.32 & & $0.071-0.65$ & $4,384-40,011$ \\
\hline
\end{tabular}


In general, despite differences in the mass and damping parameters, the cross-flow amplitude from the present study is reasonably comparable to that from aforementioned researchers. The galloping rate in the Nemes et al. [11] and $\mathrm{Xu}-\mathrm{Xu}$ et al. [14] tests are, however, slightly higher than that in the current study. This might be caused by the differences in the massdamping ratios [14] or different experimental conditions [11]. Xu-Xu et al. [14] reported that the slope of the galloping in the square cylinders varies depending on the mass ratio. It is worth noting that for the velocity ranges tested in the current study, the maximum reduced amplitude is about $1.45 \mathrm{D}$.

The lift force coefficients of the two configurations are compared in the second row of Figure 2. With the diamond cylinder, $C_{\text {Lrms }}$ discloses a sharp peak (Figure 2(c)). Here, the maximum $C_{\text {Lrms }}$ is 1.42 and occurs at $U_{r} \approx 4$. Beyond the peak, $C_{\text {Lrms }}$ falls very rapidly. Figure 2(d) shows that the maximum $C_{\text {Lrms }}$ with the square cylinder (1.09) is smaller than those for the diamond cylinder. It occurs at $U_{r} \approx 5.1$. Beyond the peak, in contrast to the diamond cylinder, $C_{\text {Lrms }}$ keeps high values. The residue at large reduced velocities stay markedly higher than those with the diamond cylinder. Large $C_{\text {Lrms }}$ at high reduced velocities is the likely drive for the galloping in the square cylinder.

Figure 2(e) shows that up to $V_{r} \approx 6$, the phase angle $\left(\Phi^{\circ}\right)$ in the diamond cylinder remains dominantly close to $0^{\circ}$. It then experiences a jump of around $180^{\circ}$ at $V_{r} \approx 7$. Beyond $V_{r} \approx 9.5, \Phi^{\circ}$ stays relatively close to $180^{\circ}$. The overall phase angle trend in Figures 2(e), characterizes a VIV type of response and resembles what is found for a linear forced system going through resonance. The $\approx 180^{\circ}$ jump in the phase angle was already attributed to a shedding mode transition from $2 \mathrm{~S}$ to $2 \mathrm{P}$ for the circular cylinders [33].

Figure 2(f) shows that with the square cylinder, the phase angle $\left(\Phi^{\circ}\right)$ is lacking the $\approx 180^{\circ}$ jump which characterizes the VIV type of response. The phase angle remains close to $0^{\circ}$ over the entire range of the velocity. As already noticed in Figure 2(b), the square cylinder undergoes galloping and does not show any resonance type of response. This is a likely reason for lacking the $\approx 180^{\circ}$ jump in the phase angle data. It may also indicate on a consistent shedding mode over different reduced velocities.

Figures $2(\mathrm{~g})$ and 2(h) plot the frequency ratio $\left(f^{*}\right)$ versus the reduced velocity $\left(V_{r}\right)$ in the diamond and square cylinders, respectively. The frequency response is separately extracted from i) transverse oscillation signals, ii) lift force signals and iii) pressure signals. The frequency of pressure fluctuations in the wake region can represent the frequency of vortex shedding. The $f_{A}{ }^{*}, f_{L}{ }^{*}$ and $f_{P}{ }^{*}$ in the diamond cylinder (Figure 2(g)) almost collapse together for the entire $V_{r}$ range tested. For $V_{r}<6$ they virtually move on the natural frequency of the diamond cylinder and beyond $V_{r}=6$ (vortex resonance and the onset of the upper branch) follow the Strouhal number line $(\mathrm{St}=0.167)$. Unlike the circular cylinders, for the resonance condition in the diamond cylinder the vortices shed at the Strouhal number and the cylinder vibrates at the same frequency. Therefore, it can be concluded that high amplitudes in the diamond cylinder fall short in controlling the vortex shedding frequency and the vortices shed control the cylinder vibrations.

With the square cylinder (Figure $2(\mathrm{~h})) f_{A}{ }^{*}, f_{L}{ }^{*}$ and $f_{P}{ }^{*}$, also nearly collapse together for the entire $V_{r}$ range tested. For $V_{r}<4.5, f^{*}$ follows the $\mathrm{St}=0.13$ line, except for few jumps to $f^{*}=1$ line. With further increase in the reduced velocity, $f^{*}$ moves almost parallel but somewhat below the natural frequency of the system.

\subsection{Hydroelastic Energy}

The hydroelastic response of different bluff bodies largely depends on the section after-body and forebody profile. Unlike the typical circular cross-sections, the separation points with the sharp edge bluff bodies are fixed at the sharp corners. Therefore, the fluid dynamic characteristics of the sharp edge crosssections strongly depend on its orientation relative to the approaching flow. The square cross-section (as typical sharp edge body), depending on the flow incidence angle, would undergo both VIV and galloping types of instabilities. In contrast, the circular cross-sections have omni-directional hydroelastic behavior and regardless of the flow incidence angle, display resonant responses.

Depending on the FIV type of the response; the mechanical power of the system and consequently the hydroelastic efficiency of the converter become different. Comparing the resonant oscillators (the diamond and circular bluff bodies), the inclined sharp edge after-body of the diamond cylinder increases the flow field strains at the wake region. This leads to much concentrated vortices with higher vorticity [11]. Thus, in places with unidirectional flow field, the diamond cross-section has advantages over circular bodies in excavating the hydroelastic energy.

In this section, the hydroelastic energy of the square cross-section in two different configurations is presented and discussed. The cross-sectional elongation ratios in both tested configurations are equal to unity.

Hydroelastic efficiency or the system energy transfer ratio is measured by the ratio of the generated mechanical power $\left(P_{\text {Mech }}\right)$ to the fluid power $\left(P_{w}\right)$ :

$$
\eta_{h e}=\frac{P_{\text {Mech }}}{P_{w}}
$$

The fluid power acting on a cylinder is assumed to be the kinetic energy of the flow over the projected area of the cylinder $(D H)$ :

$$
P_{w}=1 / 2 \rho V^{3} D H
$$



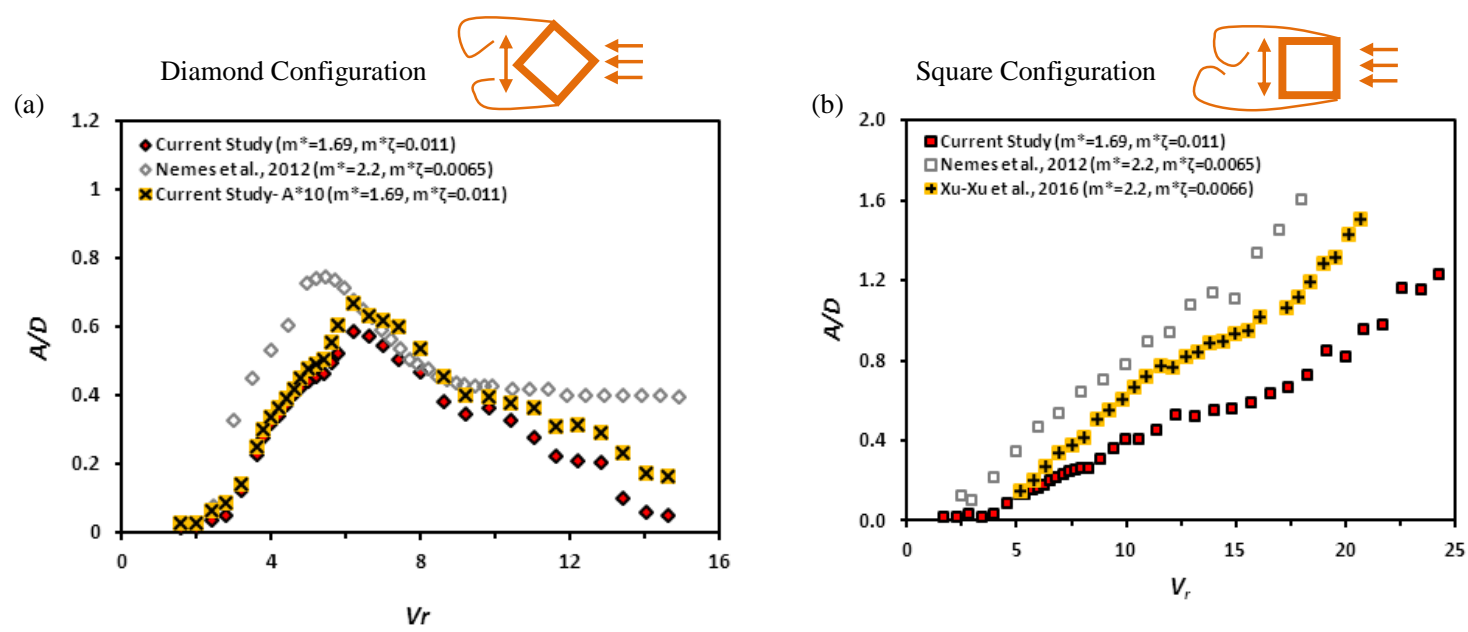

(c)

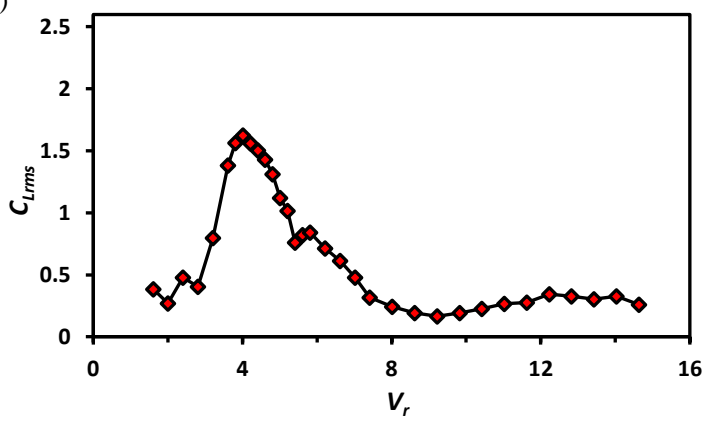

(d)
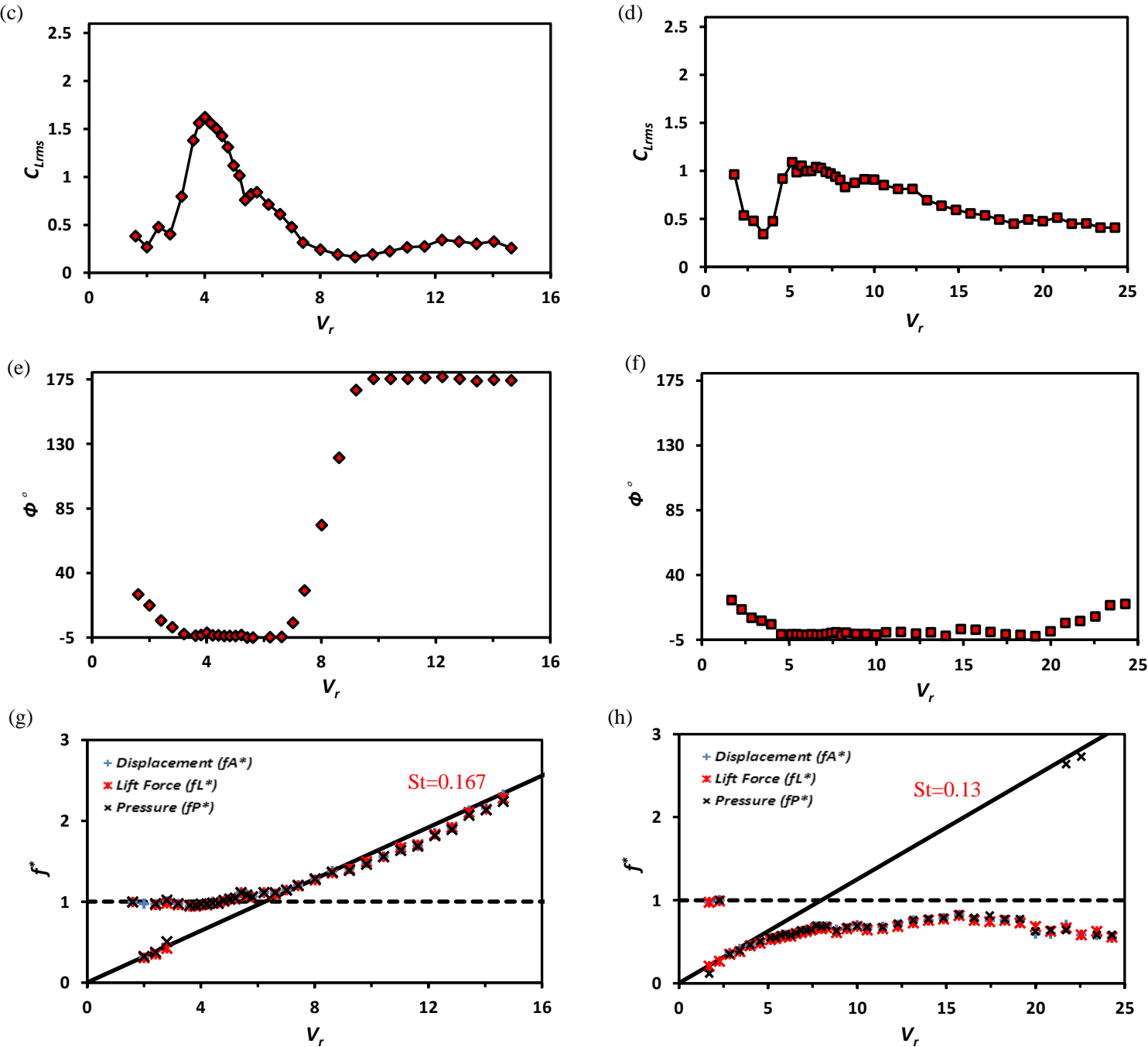

Figure 2. FIV response of the square cross-section in diamond (left) and square (right) configurations. (a,b) Response amplitudes, $(c, d)$ lift force coefficients, $(e, f)$ phase angles and $(\mathrm{g}, \mathrm{h})$ frequency ratios.

The generated mechanical power is the mechanical work $\left(W_{M e c h}\right)$ done by the oscillating cylinder duration a vibration cycle $(T)$ :

$$
P_{M e c h}=\frac{W_{M e c h}}{T}
$$

The mechanical work done by the cylinder is the force applied on the cylinder cross the cylinder's displacement. Assuming harmonic motion and harmonic forcing, the mechanical work can be simplified to Eq. 4 [18]:

$$
W_{M e c h}=\int_{0}^{T} C \dot{y}^{2} d t
$$

The harvested mechanical power from the square cross-section in two different configurations is shown in Figures 3(a) and 3(b). The figure shows that the overall mechanical work done by different oscillators is proportional to their corresponding transverse response plot (Figures 2(a) and 2(b)). The mechanical power of the diamond cylinder shows a peak. It initially increases to its maximum at $V_{r}=6$ and then gradually 
reduces to nearly zero at $V_{r}=15$. With the square cylinder, the mechanical power increases, almost monotonically, with the increase in the flow velocity.

According to the Figures 3(a) and 3(b), the maximum power obtained in the unit length of the square crosssection is $80 \mathrm{~mW} / \mathrm{m}$ and is extracted by the diamond configuration with VIV type of response. The maximum power obtained by the square configuration is $66 \mathrm{~mW} / \mathrm{m}$ which is only $17 \%$ lower than that of the diamond configuration. However, the corresponding available fluid power with the square $(10.9 \mathrm{~W} / \mathrm{m})$ is almost 11 times larger than that of the diamond cylinder $(0.9 \mathrm{~W} / \mathrm{m}$ - see Figures 3(c) and 3(d)). This is because the maximum power obtained by the square configuration is at around $157 \%$ higher flow velocity

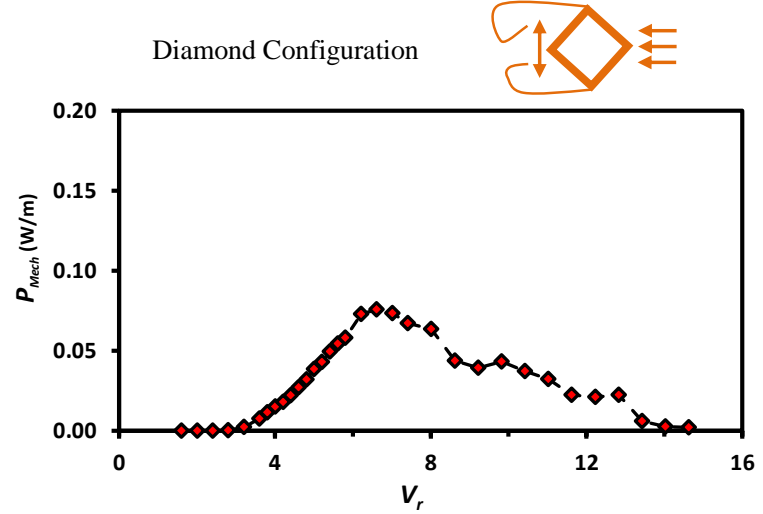

(c)

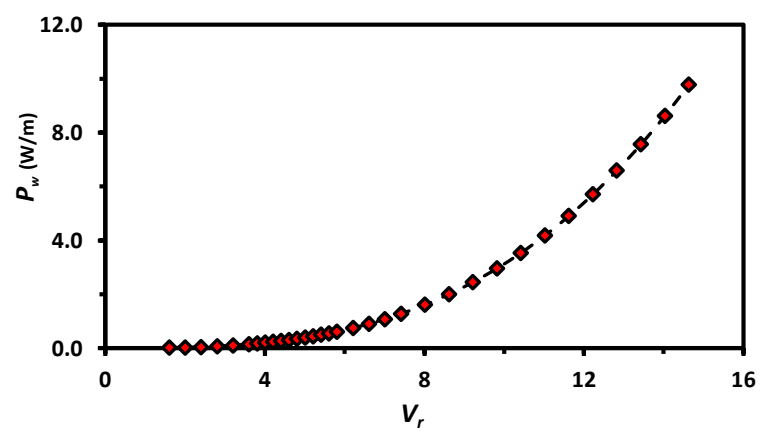

(e)

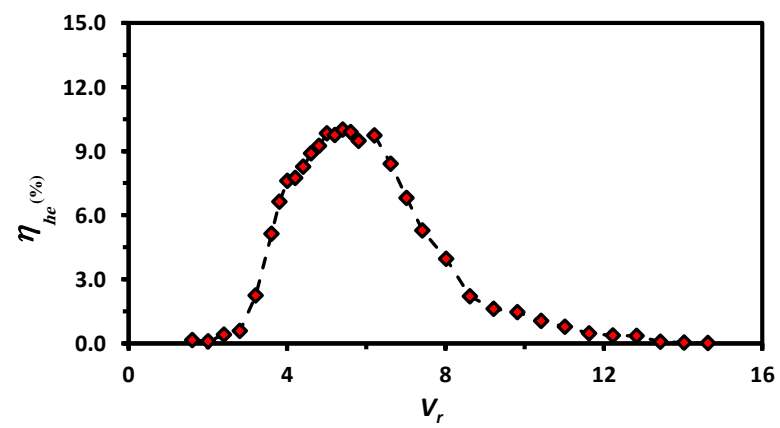

( $V=0.85 \mathrm{~m} / \mathrm{s}$ ) compared to that of the diamond cylinder $(V=0.33 \mathrm{~m} / \mathrm{s})$.

Figures 3(e) and 3(f) show the efficiency of the harvester with the square cross-section. The overall efficiency trend with the square and diamond configurations are roughly proportional to their corresponding response amplitude plot. With the diamond configuration there is a rise and fall for $3<V_{r}<13$. With the square configuration, however, the hydroelastic efficiency almost reaches a plateau for $V_{r}>7$. The maximum efficiency of the diamond cylinder is $5.61 \%$ which is around 5 times greater than the maximum efficiency of the square cylinder $(0.95 \%)$.

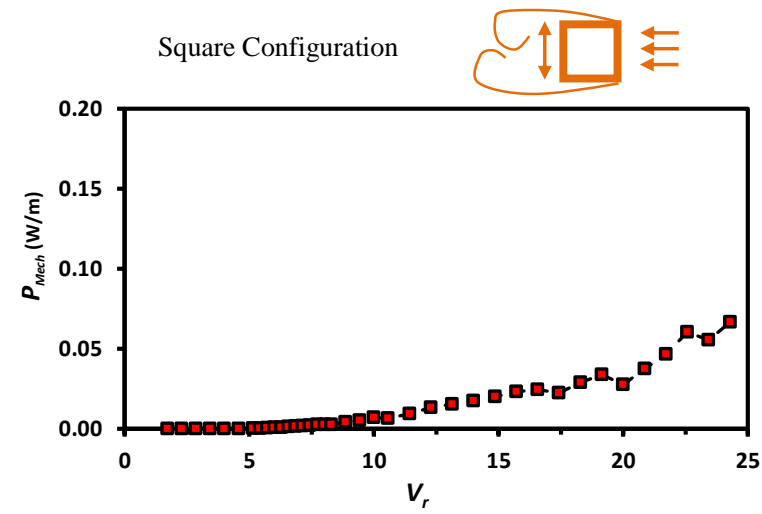

(d)

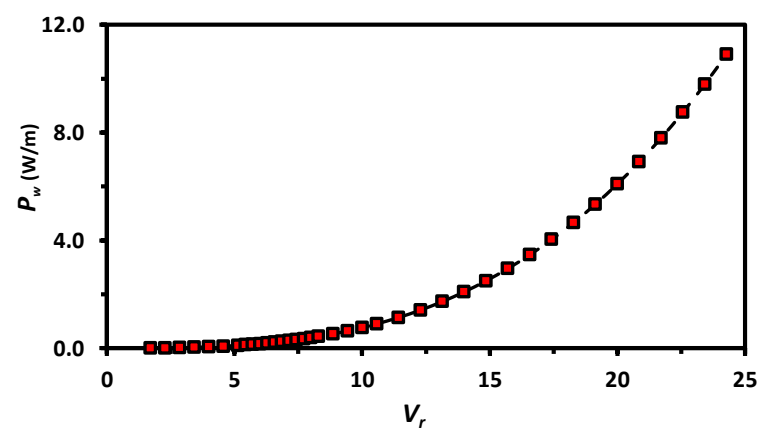

(f)

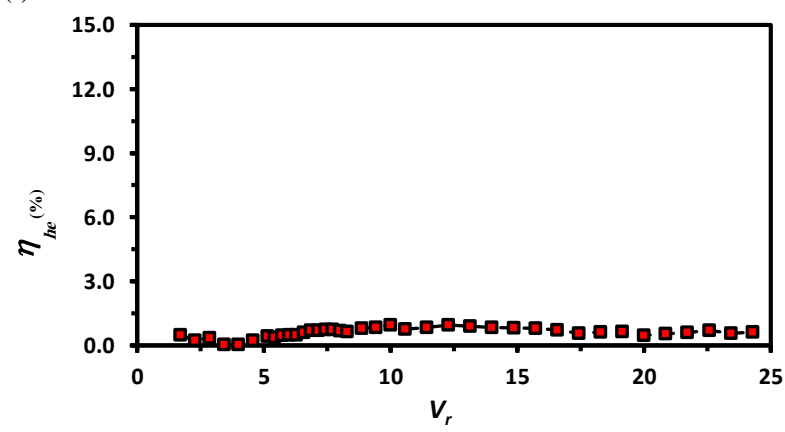

Figure 3. (a,b) mechanical power; (c,d) fluid flow power; (e,f) energy transfer ratios of the square cross-section in diamond (left) and square (right) configurations.

Table 2. Energy performance of the square cross-section in square and diamond configurations.

\begin{tabular}{lcccccc} 
Sonfiguration & $\begin{array}{c}\text { Response } \\
\text { Type }\end{array}$ & $\begin{array}{c}P_{\text {Mech-Max }} \\
(\mathrm{mW} / \mathrm{m})\end{array}$ & $\begin{array}{c}P_{\text {Mech-Avg }} \\
(\mathrm{mW} / \mathrm{m})\end{array}$ & $\begin{array}{c}\eta_{\text {he-Max }} \\
(\%)\end{array}$ & $\begin{array}{c}\eta_{\text {he-Avg }} \\
(\%)\end{array}$ \\
\hline Siamond & Galloping & 66 & 14.3 & 0.95 & 0.6 \\
\hline
\end{tabular}


For better comparison, the average values of the system mechanical power and efficiency are presented along with their corresponding maximum values in Table 2. The table shows that the maximum harvested mechanical power by the diamond configuration $\left(P_{\text {Mech }}\right.$ ${ }_{M a x}=76 \mathrm{~mW} / \mathrm{m}$ ) is close to that harvested by the square configuration $\left(P_{\text {Mech-Max }}=66 \mathrm{~mW} / \mathrm{m}\right)$. However, the averaged harvested mechanical power by the diamond cylinder is more than two times of that in the square configuration. Comparing the hydroelastic efficiencies, the differences become greater. The average transfer ratio $\left(\eta_{h e-A v g}\right)$ of the diamond cylinder is $4.5 \%$. This is around 6.5 times larger than that of the square cylinder $\left(\eta_{\text {he-Avg }}=0.6 \%\right)$. This should be added to the aforementioned 5 times larger peak transfer ratio of the diamond configuration over the square cylinder. The distinct greater efficiency of the diamond cylinder in comparison to the square configuration plus $109 \%$ higher averaged mechanical power extracted by the diamond cylinder prove the absolute advantages of VIV diamond oscillator over square galloping harvester.

\section{Conclusions}

The in-water FIV response and hydroelastic energy performance of a square cross-section were experimentally investigated. The square section was studied in two different configurations with respect to the flow direction. The experimental model was validated by comparing the FIV responses of the cylinders from the current study against the relevant published test data from other researchers. In general, the comparison between the two sets of results showed an acceptable level of correlation. When the flow was perpendicular to the side walls of the square section, the responses were of galloping type. With the galloping, the response amplitudes increased linearly with the rise in flow velocity, the lift force coefficient remained large at high flow rates and the force and displacements were in phase with the entire range of velocities tested. With 45 degrees rotation in the cylinder orientation (diamond configuration), the cylinder demonstrated resonance responses with a relatively sharp peak in the lift force and transverse response plots. The results showed that the overall mechanical work done by different oscillators is proportional to their corresponding transverse response curves. The general energy performance of the diamond VIV harvester was higher than the square galloping vibrator. Despite comparable mechanical energy harvested by the two configurations, the energy efficiency of the diamond cylinder was 5 times greater than the square configuration.

\section{Nomenclature}

$$
T=\frac{1}{f_{V}}
$$

Period of oscillations

$$
\begin{array}{ll}
\mathrm{Re}=\frac{V \quad D}{v} & \text { Reynolds number } \\
\mathrm{St}=\frac{f_{S} D}{V} & \text { Strouhal number } \\
U_{r}=\frac{1}{\mathrm{St}} & \text { Vortex resonance velocity } \\
V_{r}=\frac{V}{D \quad f_{N}} & \text { Reduced velocity } \\
m^{*}=\frac{M}{m_{d}} & \text { Mass ratio } \\
f^{*} & \text { Frequency ratio }=\frac{f}{f_{N}}
\end{array}
$$

\begin{tabular}{|c|c|c|c|}
\hline$A$ & $\begin{array}{l}\text { Transverse displacement } \\
\text { amplitude }\end{array}$ & $C$ & $\begin{array}{l}\text { Mechanical system } \\
\text { damping coefficient }\end{array}$ \\
\hline$D$ & $\begin{array}{l}\text { Characteristic length of } \\
\text { the cross-section }\end{array}$ & $K$ & $\begin{array}{l}\text { Mechanical system } \\
\text { stiffness }\end{array}$ \\
\hline$A / D$ & $\begin{array}{l}\text { Reduced transverse } \\
\text { amplitude }\end{array}$ & $M$ & Total oscillating mass \\
\hline$\dot{y}$ & Instantaneous velocity & $m_{d}$ & Displaced mass \\
\hline$f_{s}$ & Shedding frequency & $\zeta$ & System damping ratio \\
\hline$f_{N}$ & $\begin{array}{l}\text { In-water natural } \\
\text { frequency of the model }\end{array}$ & $m^{*} \zeta$ & Mass-damping \\
\hline$f$ & Vibration frequency & $P_{M e c h}$ & Mechanical power \\
\hline$H$ & Submerged length & $P_{w}$ & Fluid power \\
\hline$V$ & Flow velocity & $W_{\text {Mech }}$ & Mechanical work \\
\hline$t$ & Time & $\eta_{h e}$ & $\begin{array}{l}\text { Hydroelastic } \\
\text { efficiency }\end{array}$ \\
\hline$\rho$ & Fluid density & & \\
\hline
\end{tabular}

Where:

\section{References}

1- Fontaine, E., Morel, J.P., Damy, G., Repecaud, M., Stassen, Y., Molin, B., De Langre, E., (2003), VIV on risers with top-tensioning buoyancy-cans. Part 1: Numerical modelling and simplified analysis, Proceedings of the Thirteenth International Offshore and Polar Engineering Conference, Honolulu, Hawaii, USA, May 25-30.

2- Blevins, R., (2004), Model for Forces on and Stability of a Cylinder in a Wake, Proc Flow Induced Vibr Conf., E de Langre, ed, Ecole Polytechnique, Paris.

3- Williamson, C.H.K., Govardhan, R.N., (2004), Vortex-induced vibrations. Annual Review, Journal of Fluid Mechanics, 36:413-455.

4- Bearman, P.W., (1984), Vortex shedding from oscillating bluff bodies. Annual Review, Journal of Fluid Mechanics, 16:195-222.

5- Parkinson, G.V., (1989), Phenomena and modelling of flow-induced vibrations of bluff bodies, Progress in Aerospace Sciences, 26:169-224.

6- Sarpkaya, T., (2004), A critical review of the intrinsic nature of vortex-induced vibrations, Journal of Fluids and Structures, 19:389-447.

7- Gabbai, R.D. and Benaroya, H., (2005), An overview of modelling and experiments of vortexinduced vibration of circular cylinders, Journal of Sound and Vibration, 616:282-575. 
8- Blevins, R.D., (1990), Flow-Induced Vibration, 2nd edn, Van Nostrand Reinhold.

9- Naudascher, E. and Rockwell, D., (2005), Flowinduced vibrations: an engineering guide, Dover.

10- Païdoussis, M.P., Price, S. and De Langre, E., (2010), Fluid-Structure Interactions: Cross-FlowInduced Instabilities, Cambridge University Press.

11- Nemes, A., Zhao, J., Lo Jacono, D., Sheridan, J., (2012), The interaction between flow-induced vibration mechanisms of a square cylinder with varying angles of attack, Journal of Fluid Mechanics, 102-130.

12- Barrero-Gil, A. and Fernandez-Arroyo, P., (2013), Maximum vortex-induced vibrations of a square prism, Wind and Structures, 16(4):341-354.

13- Zhao, J., Leontini, J.S., Lo Jacono, D. and Sheridan, J., (2014), Fluid-structure interaction of a square cylinder at different angles of attack, Journal of Fluid Mechanics, 747:688-721.

14- Xu-Xu, J., Barrero-Gil, A., Velazquez, A., (2016), Dual mass system for enhancing energy extraction from Vortex-Induced Vibrations of a circular cylinder, International Journal of Marine Energy, 16:250-261.

15- Obasaju, E.D., Ermshaus, R. and Naudascher, E, (1990), Vortex-induced streamwise oscillations of a square-section cylinder in a uniform stream, J. Fluid Mech, 213:171-189.

16- Dutta, S., Panigrahi, P.K. and Muralidhar, K., (2008), Experimental investigation of flow past a square cylinder at an angle of incidence, Journal of Engineering Mechanics,134:788-803.

17- Bernitsas, M.M. and Raghavan, K., (2004), Converter of Current/Tide/Wave Energy, Provisional Patent Application, U.S. Patent and Trademark Office, Serial No. 60/628,252.

18- Bernitsas, M.M., Raghavan, K. and Ben-Simon, Y., (2008), Vivace (vortex induced vibrationaquatic clean energy): A new concept in generation of clean and renewableenergy from fluid flow, J. Offshore Mech. Arct. Eng. Trans. ASME, 130 041101:1-15.

19- Chang, C.C., Kumar, R.A. and Bernitsas M.M., (2011), VIV and galloping of single circular cylinder with surface roughness at $3.0 \times 10^{4} \leq R e \leq 1.2 \times 10^{5}$, Ocean Eng., 38-16, 1713-1732.

20- Park, H., Bernitsas, M.M. and Kumar, R.A., (2013), Enhancement of flow-induced motion of rigid circular cylinder on springs by localized surface roughness at $3.0 \times 10^{4}<R e<1.2 \times 10^{5}$, Ocean Eng., 72:403-15.

21- Kim, E.S. and Bernitsas, M.M., (2016), Performance prediction of horizontal hydrokinetic energy converter using multiple-cylinder synergy in flow induced motion, Applied Energy, 170: 92-100.

22- Kim, E.S., Bernitsas, M.M. and Kumar R.A., (2013), Multicylinder Flow-Induced Motions: Enhancement by Passive Turbulence Control at $28,000<\operatorname{Re}<120,000$, Journal of Offshore Mechanics and Arctic Engineering, Vol. 135 /021802-1.
23- Nishi, Y., Ueno, Y., Nishio, M., Quadrante, L.A.R., Kokubun, K., (2014), Power extraction using flowinduced vibration of a circular cylinder placed near another fixed cylinder, Journal of Sound and Vibration, 333: 2863-2880.

24- Abdelkefi, A., Hajj, M.R. and Nayfeh, A.H., (2013), Piezoelectric energy harvesting from transverse galloping of bluff bodies, Smart Mater. Struct., 22:015014 (11pp).

25- Zhang, J., Xu, G., Liu, F., Lian, J. and Yan, X., (2016), Experimental investigation on the flow induced vibration of an equilateral triangle prism in water, Applied Ocean Research,61:92-100.

26- Hémon, P., Amandolese, X. and Andrianne, T., (2017), Energy harvesting from galloping of prisms: A wind tunnel experiment, Journal of Fluids and Structures, 70:390-402.

27- Zeinoddini, M., Tamimi, V. and Bakhtiari, A., (2014), WIV response of tapered circular cylinders in a tandem arrangement: An experimental study, Applied Ocean Research, 47:162-173.

28- Zeinoddini, M., Tamimi, V. and Seif, M.S., (2013), Stream-wise and cross-flow vortex induced vibrations of single tapered circular cylinders: an experimental study, Applied Ocean Research, 42:124-35.

29- Tamimi, V., Naeeni, S.T.O. and Zeinoddini, M., (2017), Flow induced vibrations of a sharp edge square cylinder in the wake of a circular cylinder, Applied Ocean Research, 66:117-130.

30- Assi, G.R.S., (2009), Mechanisms for flow-induced vibration of interfering bluff bodies, $\mathrm{PhD}$ thesis, Imperial College London, London, UK.

31- Morse, T.L., Govardhan, R.N. and Williamson, C.H.K., (2008), The effect of end conditions on the vortex-induced vibration of cylinders, Journal of Fluids and Structures, 24:1227-39.

32- Blevins, R.D. and Coughran, C.H.S., (2009), Experimental investigation of vortex-induced vibration in one and two dimensions with variable mass, damping, and Reynolds number, Journal of Fluids Engineering, Vol. 131/101202-1.

33- Khalak, A. and Williamson, C.H.K., (1999), Motions, forces and mode transitions in vortexinduced vibrations at low mass-damping, Journal of Fluids and Structures,13:813-51. 